\title{
JAMES, Harold, Die Deutsche Bank und die « Arisierung 》)
}

Hervé Joly

\section{OpenEdition}

\section{Journals}

Édition électronique

URL : http://journals.openedition.org/ifha/1216

DOI : $10.4000 /$ ifha. 1216

ISSN : 2198-8943

Éditeur

IFRA - Institut franco-allemand (sciences historiques et sociales)

Référence électronique

Hervé Joly, "JAMES, Harold, Die Deutsche Bank und die "Arisierung » », Revue de l'IFHA [En ligne], Date de recension, mis en ligne le 01 janvier 2002, consulté le 22 septembre 2020. URL : http:// journals.openedition.org/ifha/1216; DOI : https://doi.org/10.4000/ifha.1216

Ce document a été généré automatiquement le 22 septembre 2020

(CIFHA 


\title{
JAMES, Harold, Die Deutsche Bank und die « Arisierung "
}

\author{
Hervé Joly
}

Cette étude sur l'implication de la Deutsche Bank dans la politique de spoliation des Juifs (" aryanisation ») constitue le deuxième rapport, après celui de Jonathan Steinberg sur les transactions d'or publié deux ans auparavant, issu des travaux de la commission de cinq historiens constituée en 1998 par la plus grande banque allemande pour étudier son activité sous le nazisme. Il est, comme le précédent, signé d'un seul de ses membres, le professeur de Princeton H.J., l'ensemble de la commission indiquant dans un préambule que les autres membres se sont contentés « de lire attentivement et de discuter intensément " le texte. Ils indiquent que leurs appréciations divergeaient sur les responsabilités de Hermann Josef Abs, jeune dirigeant de la Deutsche Bank sous le nazisme qui deviendra le banquier le plus influent de la RFA. Par ailleurs, s'ils se sont accordés sur l'impossibilité de fournir un montant absolu des gains réalisés grâce aux spoliations, ils évaluent de manière variable l'importance relative de ces gains pour la banque.

H.J. avait déjà, dans le cadre d'un ouvrage collectif publié en 1995 à l'occasion du 125ème anniversaire de la Deutsche Bank, écrit un long chapitre qui, pour la première fois dans le cadre d'un tel travail de commande, apportait un regard critique sur l'histoire de la banque sous le nazisme. Cette seconde étude bénéficie de l'apport de sources nouvelles, telles que les archives des différentes succursales, qui permettent d'analyser comment l'« aryanisation "a été menée de manière différenciée au plan local, ou des fonds provenant des anciens pays communistes. On peut simplement s'étonner que la rigidité de la législation allemande sur les archives publiques ne permette pas, dans le cadre d'un tel projet, d'obtenir une dérogation pour consulter les fonds des services fiscaux, disponibles seulement après 80 ans.

H.J. examine successivement la politique menée par la Deutsche Bank dans les frontières de l'Allemagne de 1937, puis à l'étranger dans les territoires annexés ou occupés à partir de 1938, en Autriche, en Tchécoslovaquie et en Pologne en particulier. Sur le territoire allemand, il montre d'abord que la Deutsche Bank a elle-même été 
confrontée à des attaques antisémites, dans la mesure où plusieurs de ses dirigeants importants en 1933 étaient d'origine juive. Ceux-ci ont été plus ou moins rapidement écartés après 1933, avant même que cela devienne une obligation légale, la banque cédant aux pressions nazies. H.J. insiste cependant sur le fait que cette bonne volonté à l'égard du régime n'a pas empêché que la banque reste, comme grand institut capitaliste, suspecte aux yeux des nazis d'être un vecteur d'influence juive. Elle a donc dû se battre pour, aux côtés notamment de petites banques régionales ou des caisses d'épargne mieux en cour auprès du régime, prendre sa place dans la politique d'" aryanisation ". Elle s'est cependant rapidement imposée comme un acteur incontournable, au moins pour les grandes affaires, grâce en particulier à l'importance de ses réseaux à l'étranger. Les modalités d'intervention de la Deutsche Bank ont été très diverses. Une distinction chronologique est d'abord faite, selon qu'il s'agissait simplement dans les premières années du régime pour des entreprises considérées comme juives de répondre à des pressions antisémites informelles qui les mettaient en difficulté, notamment pour l'accès aux marchés publics, ou, à partir de 1937-1938, d'appliquer une politique de spoliation généralisée. La Deutsche Bank est ensuite amenée à intervenir dans des circonstances différentes : elle est parfois sollicitée par les dirigeants ou actionnaires juifs de l'entreprise concernée, qui cherchent à céder dans les meilleures conditions possibles une affaire qu'il leur devient impossible de conserver. Elle peut aussi intervenir à la demande du régime pour faciliter une " aryanisation " rendue difficile par l'implication d'actionnaires domiciliés à l'étranger. Elle peut enfin être saisie par un acquéreur potentiel qui cherche à obtenir les moyens financiers nécessaires. L'appréciation portée sur son comportement dépend donc largement de ces circonstances. De plus, il faut distinguer les importantes affaires gérées au niveau central par la direction de la banque des petites menées, avec une importante autonomie, par les différentes succursales locales. L'ouvrage retrace ainsi une succession de cas, sans que les conclusions tirées permettent de se faire une opinion très claire. L'appréciation des bénéfices réalisés est également difficile : les commissions normales prévues pour les opérations sont assez faibles, mais certaines circonstances (conservation un temps en portefeuille d'actions en forte hausse dans l'intervalle par exemple) peuvent générer des profits importants.

Le bilan est beaucoup moins nuancé dans les territoires conquis. L'action de la Deutsche Bank s'inscrit inévitablement dans le cadre d'une politique globalement prédatrice, qui ne laisse aucune place pour les égards aux victimes. Les bénéfices financiers ne sont là encore pas forcément considérables. Il s'agit d'abord pour la première banque allemande de tenir son rang, face à un challenger, la Dresdner Bank, très actif. L'enjeu amène donc la banque à se compromettre dans les pires dimensions criminelles du régime.

Par la richesse des sources utilisées et la précision de ses analyses, le livre fournit une description très détaillée de l'implication de la Deutsche Bank dans cette étape essentielle vers l'extermination finale que constituait la spoliation. On peut simplement regretter que le style du livre et son écriture souvent impersonnelle l'apparentent trop à un rapport administratif. On aurait pu attendre un engagement plus ferme de l'auteur dans ses conclusions.

Hervé JOLY 